\title{
Mortality after cancer diagnosis in HIV-infected individuals treated with antiretroviral therapy
}

\author{
Chad J. Achenbach ${ }^{\mathrm{a}}$, Stephen R. Cole ${ }^{\mathrm{b}}$, Mari M. Kitahata ${ }^{\mathrm{c}, \mathrm{d}}$, Corey Casper ${ }^{\mathrm{c}, \mathrm{d}, \mathrm{e}, \mathrm{f}}$, James H. \\ Willig $^{g}$, Michael J. Mugavero ${ }^{9}$, and Michael S. Saag ${ }^{g}$ \\ aDivision of Infectious Diseases, Center for Global Health, Feinberg School of Medicine, \\ Northwestern University, Chicago, Illinois \\ bDepartment of Epidemiology, Center for AIDS Research, Gillings School of Global Public Health, \\ University of North Carolina at Chapel Hill, Chapel Hill, North Carolina \\ 'Department of Medicine, University of Washington, Seattle, Washington \\ ${ }^{d}$ Center for AIDS Research, University of Washington, Seattle, Washington \\ eDepartment of Epidemiology, University of Washington, Seattle, Washington \\ fVaccine and Infectious Diseases Division, Fred Hutchinson Cancer Research Center, Seattle, \\ Washington \\ 9Department of Medicine, Center for AIDS Research, University of Alabama at Birmingham, \\ Birmingham, Alabama, USA
}

\section{Abstract}

Objectives-To evaluate survival and predictors of mortality after cancer diagnosis among HIVinfected persons receiving combination antiretroviral therapy (cART).

Design-Multisite cohort study.

Methods-We examined all-cause mortality among HIV-infected patients treated with cART in routine care at eight US sites and diagnosed with cancer between 1996 and 2009, and predictors of mortality using Cox proportional hazards regression models. Non-AIDS-defining cancers (NADCs) were classified as related and unrelated to viral coinfections.

Results-Out of 20677 persons in the Centers for AIDS Research Network of Integrated Clinical Systems cohort, 650 cART-treated individuals developed invasive cancer. Of these, 305 died during 1480 person-years of follow-up; crude mortality rate was 20.6 per 100 person-years [95\% confidence interval (CI) 18.4, 23.1] and overall 2-year survival was 58\% (95\% CI 54, 62). Highest mortality was seen in primary central nervous system non-Hodgkin's lymphoma, liver, and lung cancer with rates of 90.6, 84.3, and 68.1 per 100 person-years, respectively. Adjusted hazard of death was higher among those who were older and had stage IV cancer. Adjusted hazard of death was lower among those with higher CD4 cell counts at cancer diagnosis, who achieved 
HIV-RNA suppression ( $\$ 400$ copies/ml) on cART, received any cancer treatment, and had AIDSdefining cancer or infection-related NADCs compared to infection-unrelated NADCs.

Conclusion-Independent predictors of mortality after cancer diagnosis among HIV-infected persons include poor immune status, failure to suppress HIV-RNA on cART, cancer stage, and lack of cancer treatment. Modification of these factors with improved strategies for the prevention and treatment of HIV and HIV-associated malignancies are needed.

\section{Keywords}

antiretroviral therapy; cancer; HIV; mortality; survival

\section{Introduction}

The widespread use of combination antiretroviral therapy (cART) has led to dramatic reductions in morbidity and mortality from AIDS-related conditions for individuals infected with HIV [1,2]. However, several studies have reported an increased incidence of nonAIDS-defining cancers (NADCs) [3-14], particularly those related to oncogenic viral coinfections, such as human papilloma-virus (HPV), Epstein-Barr virus (EBV), and hepatitis $\mathrm{B}$ or $\mathrm{C}$ virus (HBV or HCV) $[8,15,16]$, among individuals with HIV-infection compared with the general population.

Trends in relative incidence rates of AIDS-defining cancers (ADCs) and NADCs have been well characterized [8,15-19], but little is known about survival after a diagnosis of cancer in the setting of HIV infection. Previous studies have been limited to examining individuals with AIDS in the pre-cART era or have lacked information regarding important risk factors [20-22]. We studied a large cohort of HIV-infected individuals engaged in routine care at eight clinical sites across the United States to define risk factors and mortality rates after a diagnosis of cancer in the modern cART era.

\section{Methods}

\section{Study population}

The Centers for AIDS Research (CFAR) Network of Integrated Clinical Systems (CNICS) was initiated in September 2006 to develop a comprehensive and standardized clinical data repository from point-of-care electronic medical record systems to support population-based HIV research [23]. The CNICS cohort includes over 20000 HIV-infected adults (at least 18 years of age) engaged in clinical care from 1 January 1995 to the present at eight CFAR sites. The frequency of follow-up averages every 3 months, however, patients can be seen more or less often depending on clinical care. CNICS is a dynamic cohort with approximately 1400 new patients enrolling and $10 \%$ of existing patients leaving care each year [23]. Institutional review boards at each University approved study protocols.

We examined all individuals in the CNICS cohort who were treated with cART for at least 6 months and had a diagnosis of cancer between 1 January 1996 and 1 July 2009. Follow-up was administratively censored at 7 years to ensure adequate numbers of patients at risk for mortality. 


\section{Sources of data}

Upon entry into the CNICS cohort, standardized demographic and historical information, including prior diagnoses and antiretroviral treatment, are collected. Once enrolled in the cohort, data are prospectively captured at outpatient and inpatient encounters at the site medical center and include medications, laboratory test results, and AIDS-defining and nonAIDS-defining conditions diagnosed by the treating provider and verified by medical record review (www.cnics.net). The following variables were included in this analysis: demographics (i.e., year of birth, sex, race/ethnicity), alcohol and tobacco exposure, intravenous drug use (IDU) as a risk factor for HIV transmission, HBV and HCV viral coinfection, AIDS-defining diagnoses, cART, CD4 cell counts, and plasma HIV-RNA levels. HCV infection was defined by positive $\mathrm{HCV}$ antibody or HCV-RNA testing prior to cancer diagnosis. HBV infection was defined by positive HBV surface antigen or HBVDNA testing prior to cancer diagnosis. CD4 cell count and HIV-RNA values at the time of cancer diagnosis were the values closest to the diagnosis date within a window of 9 months before and 3 months after cancer diagnosis. HIV-RNA suppression was defined as any HIVRNA level less than or equal to 400 copies/ml after cART initiation and before cancer diagnosis. Tobacco exposure and alcohol abuse were determined at the clinical assessment closest to cancer diagnosis to be current, past, or never. If there were no clinical records documenting alcohol or tobacco assessments prior to the cancer diagnosis or for 6 months after, the patient was classified as never exposed. Alcohol exposure was defined as abuse if qualitative assessments described 'abuse', 'daily', 'dependence', or 'binge' and if quantitative alcohol exposure was greater or equal to three drinks daily or 10 drinks per week.

\section{Cancer verification}

At each CNICS site, incident diagnoses of invasive cancer were reviewed using a standardized protocol to confirm the diagnosis and collect detailed information regarding cancer type, histology, grade, staging, and treatment from the medical record. Every CNICS site used the same protocol for cancer review and data collection. Cancer types were classified according to a predefined grouping based on location (i.e., anal, breast, cervix, and lung) and/or histopathologic reports (i.e., Kaposi's sarcoma, lymphoma, and leukemia), nonmelanoma skin cancers were not included. Histology, grade, tumor nodes and metastasis and summary staging were obtained from information provided in pathology reports and clinician notes. If a patient had more than one cancer diagnosis, we analyzed only the first cancer observed. Treatment for cancer was defined as having received at least one treatment modality regardless of timing or completion of therapy categorized as surgery, chemotherapy, radiation, biologic, or none. Biopsy results confirmed $89 \%$ of non-Kaposi's sarcoma cancers; the remaining cancers were diagnosed on the basis of clinical, radiographic, or historical information. Kaposi's sarcoma was staged as mucocutaneous or visceral on the basis of AIDS Clinical Trials Group classification tumor status. Cancers qualitatively described as 'metastatic', 'widespread', or 'disseminated' were defined as stage IV. Visceral Kaposi's sarcoma and primary central nervous system (CNS) non-Hodgkin's lymphoma (NHL) were classified as 'stage IV equivalent' and mucocutaneous Kaposi's sarcoma was classified as 'not stage IV equivalent'. Cancer stage was categorized as 
missing/unknown if staging information was not available after careful review of medical records.

To address the lack of complete data on cancer staging, we imputed missing 'stage IV equivalent' information as per the methods described below for the final multivariable analysis of all cancer cases. To examine the validity of this approach, we performed two prespecified subset analyses in which stage of cancer was known for all cases. The first subset analysis examined cancers using the I-IV summary staging classification (excluding Kaposi's sarcoma, leukemia, multiple myeloma, primary CNS NHL, and primary brain). In this subset, we ran the same multivariable analyses conducted for the full cohort of cancer cases with the variable 'stage IV' or 'not stage IV'. For the second subset analysis, we performed the same multivariable analysis with the addition of cases categorized as 'stage IV equivalent' or 'not stage IV equivalent'.

ADCs included cervical, Kaposi's sarcoma, and NHL according to the 1993 Centers for Disease Control and Prevention criteria [24]. NADCs were classified into those related to viral coinfections (HPV, EBV, and viral hepatitis) and those unrelated to viral coinfection. Infection-related NADCs included squamous cell anal, squamous cell oral cavity/pharynx, Hodgkin's lymphoma, liver with viral hepatitis, vagina/vulva, and penis [16]. Infectionunrelated NADCs included nonsquamous cell anal, biliary, bladder/urinary, primary brain, breast, colorectal, esophagus, nonsquamous cell oral cavity/pharynx, other head and neck, ovary, other (type unspecified or unknown), pancreas, kidney, leukemia, liver without viral hepatitis, lung, multiple myeloma, melanoma, peritoneum/retroperitoneum, prostate, small intestine, soft tissue, stomach, testicular, thyroid, trachea/pleura, and uterus.

\section{Mortality ascertainment}

Death data were obtained from clinic sources and confirmed by the National Death Index. In addition, the National Death Index was searched quarterly for all CNICS participants who were not known to be alive. We examined rates and predictors of all-cause mortality.

\section{Statistical analysis}

Mortality rates were calculated as number of deaths per 100 person-years of follow-up time. The complement of Kaplan-Meier survival curves were used to display the time from cancer diagnosis to death [25], as well as to estimate 2-year survival. We used Cox proportional hazards regression models to examine predictors of mortality [26]. Factors included in multivariable models were type of cancer (categorized as ADC, infection-related NADC, and infection-unrelated NADC), age, race/ethnicity, sex, viral hepatitis coinfection, current and past tobacco exposure, current and past alcohol abuse, nadir CD4 cell count, CD4 cell count at cancer diagnosis, pre-cART HIV-RNA level, HIV-RNA suppression on cART, noncancer AIDS-defining diagnosis, cancer stage, cancer treatment, and year of cancer diagnosis. The assumption that continuous predictors (i.e., age at cancer diagnosis, CD4 cell count, and HIV-RNA) had a log-linear association with the mortality hazard was explored and when violated the continuous predictor was categorized to reflect the nonlog-linear association. The proportional hazards assumption was explored graphically by plotting the $\log$ of the cumulative hazard by time. 
We imputed the median or modal value for infrequently missing variables (see Table 1, footnote b). In the final multivariable model, for cases missing 'stage IV equivalent' information (see Table 4, footnote b), we imputed missing values 25 times using a Markov chain Monte Carlo algorithm as implemented in the SAS (SAS Institute Inc., Cary, North Carolina, USA) procedure multiple imputation [27,28]. The first 1000 Monte Carlo draws were discarded to allow for a burn-in period, and the subsequent 5000 draws were used to summarize the joint posterior density. Imputations were based on information from all variables in Tables 1 and 4, including the occurrence and timing of deaths. We estimated summary hazard ratios as the antilog of the average of the $25 \log$ hazard ratios. We estimated the variance of the hazard ratios by Rubin's canonical imputation variance formula [27].

\section{Results}

Among a total of 20677 HIV-infected persons enrolled in the CNICS cohort, there were 1454 incident cases of invasive cancer. Six hundred and fifty patients treated with cART had a subsequent diagnosis of cancer between 1996 and 2009 (Table 1). The median age at cancer diagnosis was 44 years, $52 \%$ of participants were white, $86 \%$ were men, $18 \%$ reported IDU as a risk factor for transmission of HIV-infection, $21 \%$ were coinfected with viral hepatitis, $38 \%$ were current smokers ( $24 \%$ former), and $15 \%$ currently abused alcohol ( $8 \%$ former). Prior to treatment with cART, participants had a median nadir CD4 cell counts of 45 cells/ $\mu \mathrm{l}$ and a median HIV-RNA of $5.4 \log _{10}$ copies $/ \mathrm{ml}$. The initial cART regimen was anchored with an unboosted protease inhibitor in 269 of 650 (41\%) of patients, a ritonavirboosted protease inhibitor in 197 of 650 (30\%), a nonnucleoside reverse transcriptase inhibitor (NNRTI) in 166 of 650 (26\%), and three nucleoside reverse transcriptase inhibitors in 18 of $650(3 \%)$. The median time on cART before a diagnosis of cancer was 3.2 years. At the time of cancer diagnosis, $92 \%$ of the patients were still on cART, which included regimens anchored with a boosted protease inhibitor in 362 of $595(60 \%)$ of patients, a NNRTI in 158 of 595 (27\%), an unboosted protease inhibitor in 57 of $595(10 \%)$, and other medications in 18 of 595 (3\%). At any time before cancer diagnosis, 551 of $650(85 \%)$ of patients achieved HIV-RNA suppression to less than or equal to $400 \mathrm{copies} / \mathrm{ml}$. At cancer diagnosis, the median CD4 cell count was 204 cells/ $\mu$ and median HIV-RNA was $2.6 \log _{10}$ copies $/ \mathrm{ml}$.

Infection-unrelated NADCs comprised 34\% of cancers, whereas infection-related NADCs were $17 \%$ of cancers and ADCs accounted for $49 \%$ of cancers. Overall, the most common incident cancers were Kaposi's sarcoma (29\%), NHL (21\%), lung (9\%), and anal (8\%) (Table 2). Staging information was available for 256 of 427 (60\%) of cancers that use the IIV summary staging classification (excluding cases of Kaposi's sarcoma, leukemia, multiple myeloma, primary CNS NHL, and primary brain). Of the 256 cancers classified by summary stage, 20, 19, 15, and 46\% were stage I, II, III, and IV, respectively. Among patients with staging information, stage IV disease was documented in 40 of 70 (57\%) with NHL, 26 of $42(62 \%)$ with lung cancer, two of $32(6 \%)$ with anal cancer, 19 of $30(63 \%)$ with Hodgkin's lymphoma, two of $10(20 \%)$ with prostate cancer, four of 12 (33\%) with liver cancer, two of nine $(22 \%)$ with kidney cancer, one of four (25\%) with breast cancer, three of six (50\%) with colon cancer, zero of two (0\%) with melanoma, and 20 of $55(36 \%)$ with other cancer 
types. In addition, when classifying visceral Kaposi's sarcoma and primary CNS NHL as 'stage IV equivalent', staging information was available for $73 \%$ of cancers (463 of 634 , excluding 16 cases of leukemia, multiple myeloma, and primary brain cancer). The majority (237 of $463,52 \%)$ of these cancers were classified as 'stage IV equivalent.'

Among the $650 \mathrm{HIV}$-infected patients diagnosed with invasive cancer on cART, there were 305 deaths during 1480 person-years of follow-up for a crude mortality rate of 20.6 per 100 person-years [95\% confidence interval (CI) 18.4, 23.1], and an overall 2-year survival of $58 \%(95 \%$ CI 54, 62) (Table 3). The highest mortality rates were among those with primary CNS NHL, liver, and lung cancer with rates per 100 person-years of 90.6, 84.3, and 68.1, respectively. The adjusted hazard ratio for death was higher among those who were older and had 'stage IV equivalent' cancer (Table 4). The adjusted hazard ratio for death was lower among those who had higher CD4 cell counts at cancer diagnosis, achieved HIV-RNA suppression to less than or equal to 400 copies/ml, received cancer treatment, and had ADCs or infection-related NADCs compared with infection-unrelated NADCs (Table 4 and Fig. 1). In adjusted analyses of the subset of 256 patients with known cancer summary stage, we found similar associations between all-cause mortality and age (hazard ratio 1.26; 95\% CI $1.00 ; 1.60, P=0.05$ ), HIV-RNA suppression (hazard ratio $0.38 ; 95 \%$ CI $0.20,0.71 ; P<$ 0.01 ), noncancer AIDS diagnosis (hazard ratio 1.70; $95 \%$ CI 1.10, 2.63; $P=0.02$ ), stage IV cancer (hazard ratio 2.88; 95\% CI 1.89, 4.37; $P<0.01$ ), cancer treatment (hazard ratio 0.41; 95\% CI 0.20, 0.84; $P=0.02$ ), and infection-related NADCs (hazard ratio 0.36; 95\% CI 0.19, $0.68 ; P<0.01)$. In additional adjusted analyses of the subset of 463 patients with known 'stage IV equivalent' cancer staging, we also found similar associations between all-cause mortality and age (hazard ratio 1.47; 95\% CI 1.22, 1.77; $P<0.01$ ), HIV-RNA suppression (hazard ratio $0.28 ; 95 \%$ CI $0.18,0.44 ; P<0.01$ ), noncancer AIDS diagnosis (hazard ratio $1.41 ; 95 \%$ CI 1.00, 2.00; $P=0.05$ ), stage IV cancer (hazard ratio 2.68; 95\% CI 1.89, 3.80; $P$ $<0.01$ ), receiving cancer treatment (hazard ratio $0.49 ; 95 \% \mathrm{CI} 0.31 ; 0.79, P<0.01$ ), ADCs (hazard ratio $0.55 ; 95 \% \mathrm{CI} 0.35,0.86 ; P<0.01$ ), and infection-related NADCs (hazard ratio $0.43 ; 95 \%$ CI $0.23,0.78 ; P<0.01)$.

\section{Discussion}

Cancer has been an important cause of morbidity and mortality for individuals with HIVinfection since the beginning of the epidemic. Indeed, we found that in a large cohort of over $20000 \mathrm{HIV}$-infected persons receiving care at eight major metropolitan areas across the United States, more than $3 \%$ of patients receiving antiretroviral therapy developed cancer. We identified known risk factors for mortality including older age, stage IV cancer at time of diagnosis, and lack of cancer treatment. We also found key factors associated with mortality that are specific to HIV-infected patients and have not been previously reported, including failure to suppress HIV-RNA, low CD4 cell count at cancer diagnosis, and cancers unrelated to a viral coinfection. A possible explanation for these findings would be that HIV-RNA reduction and immunologic recovery from cART led to suppression of viral coinfection replication and its effects on tumor pathogenesis or growth of these cancers. Alternatively, among HIV-infected patients, these cancers may be less aggressive or more responsive to cancer treatment compared with NADCs unrelated to viral coinfection. However, classifying cancers as related to viral coinfection may not be of central clinical 
importance when studying outcomes such as mortality. For example, in Figure 1c, we observed a notable difference within infection-related NADCs in which mortality was considerably higher for those with liver cancer compared with anal cancer or Hodgkin's lymphoma. These findings point to the need for further study of the epidemiology and pathophysiology of cancer progression and viral coinfections in HIV disease. Particularly, more information is needed regarding outcomes of individual types of cancer among HIVinfected individuals.

We observed several important findings with regard to mortality rates for individual cancers. Consistent with reports from other HIV-infected populations, the highest mortality rates for NADCs were among patients with liver and lung cancer [20,22], accounting for nearly 50\% of deaths after NADC. Two-year survival for patients with liver cancer in this study was only $12 \%$. Previous studies of mortality after liver cancer comparing those with and without HIV-infection have been mixed [29,30]. Mortality after NHL is known to be worse for patients with HIV, although a recent study from Europe suggested improved survival with cART [31]. Our results were consistent with another recent study of survival after NHL that directly compared those with and without HIV-infection in the cART era [32] and found 40\% 2-year survival for patients with HIV and 70\% for those without HIV. Our findings also suggest that HIV-infection may increase mortality for patients with kidney cancer, as 2year survival was only 58\%. Considering immune-modulating therapies are often used for treatment of renal cell carcinoma, immune deficiency or dysregulation due to HIV infection could unfavorably alter the pathogenesis of this cancer. This finding has not been previously reported and requires further investigation.

Increased mortality observed among those with lower CD4 cell counts highlights the importance of timely HIV treatment. The majority of patients in this study had very low nadir CD4 cell counts prior to initiation of cART and were unable to adequately rebuild their immune system with treatment. This finding is consistent with several studies showing that severity of immune suppression at time of cART initiation is predictive of the ability of antiretroviral treatment to significantly increase CD4 cell count [33-36]. Ongoing inflammation and immunodeficiency in patients who initiate cART at lower CD4 cell counts increases the risk of developing comorbid conditions, as demonstrated by the prognostic value of CD4 cell count and other immunologic markers in patients treated with cART [34,37]. Our results emphasize the importance of prompt and effective treatment of HIV with cART to reduce mortality from AIDS and non-AIDS-defining comorbid conditions, such as cancer.

More than 50\% of HIV-infected patients diagnosed with cancer in our cohort had advanced (stage IV) disease at the time of diagnosis. Additionally, at least $25 \%$ of patients received no cancer treatment. This is surprising, as individuals in this study were receiving cART in specialized HIV clinics and routinely engaged in care with quarterly monitoring on average. Although it is possible that HIV infection and associated immune system dysfunction accelerate the rate of cancer progression to advanced and untreatable states, our findings could be explained by poor cancer awareness, inadequate screening practices, or lack of prompt therapy. HIV-infected individuals may require novel cancer prevention and treatment strategies that incorporate key prognostic factors such as those found in our study, 
including suppression of HIV-RNA, prevention of CD4 cell count decline, and cancer screening initiated at a younger age than in the general population. Further research is needed to define the optimal timing and modalities for cancer screening and treatment among HIV-infected populations.

This study has several limitations. First, associations presented here reflect observational evidence and, therefore, could have been influenced by unmeasured risk factors for death such as active substance abuse or other comorbid disease. Second, although the present work reflects a large study of mortality after cancer diagnosis, information regarding tobacco exposure, alcohol abuse, viral hepatitis coinfection, noncancer AIDS diagnoses, cancer stage, and cancer treatment were subject to imprecision and potential misclassification. Third, despite extensive medical record review, detailed cancer staging information was not available for $26 \%$ of cancer diagnoses, a key predictor of mortality. However, we used multiple imputations to account for missing staging information in the final adjusted model for the full cohort, and our results were consistent with multivariable analyses conducted on two subsets of patients with known cancer stage. Fourth, we did not account for changes over time for certain factors, such as CD4 cell count and cART, which will be the focus of subsequent analyses. Fifth, we did not have information on cause of death and were unable to provide direct comparisons of our findings with a matched population of patients without HIV infection. In the modern era of cART, patients with cancer and HIV infection have several competing risks for death that vary depending on individual cancer type. We were limited by a small number of cases of specific cancer types and aggregate findings may not accurately capture outcomes for less common cancers. Future research in this area will require multicenter cohorts with large numbers of individuals with specific cancer types and detailed information about cause of death.

Despite these limitations, this work has several strengths. First, this was a multicenter collaborative effort from a large and diverse population with cancer diagnoses rigorously verified to minimize misclassification. Second, the occurrence and timing of mortality was based on active and passive surveillance also leading to minimal misclassification. Third, distinct from previous studies, we were able to examine multiple risk factors for mortality among HIV-infected patients with cancer including CD4 cell count, HIV-RNA level, cancer stage, viral hepatitis coinfection, smoking, and alcohol exposure.

In conclusion, among individuals with cancer and HIV infection treated with cART, we observed independent effects on mortality of low CD4 cell count, lack of HIV-RNA suppression and cancer treatment, and cancers unrelated to infection. In addition, a large proportion of patients engaged in routine HIV care were diagnosed late with stage IV cancer. Our results support earlier initiation of cART and aggressive cancer screening and treatment practices to maintain immunologic function, obtain optimal virologic suppression, control viral coinfections, detect cancers at an early stage, and provide appropriate cancer therapies. These findings exemplify the dynamic nature of the HIV epidemic in the current era of cART and highlight the need for continued research on prevention, diagnosis, and treatment of non-AIDS-defining conditions, including cancer. 


\section{Acknowledgements}

Funding for this study was provided by NIAID with a NCI supplement (R24 AI067039) and the University of Washington AIDS and STD Research Training Grant (T32 AI07140-31).

C.J.A. worked closely with M.M.K., M.S.S., and the Centers for AIDS Research Network of Integrated Clinical Systems (CNICS) data management core at the University of Washington to design and implement the cancer data collection process. C.J.A. and S.R.C. designed the study and acquired the data from the CNICS data management core. S.R.C. selected the appropriate statistical analyses and executed them with the assistance of C.J.A. The initial draft of the manuscript was written by C.J.A. and S.R.C. M.M.K. and C.C. assisted in refining the statistical analysis and preparing subsequent drafts of the manuscript. Throughout the study, all authors participated in discussions about the design, statistical analyses, and interpretation of findings. All authors were involved in the review and editing process of the final manuscript for submission.

These findings are presented on behalf of the CNICS. The authors would like to thank all the CNICS investigators and data management teams from the eight sites who contributed to the completion of this study at Case Western Reserve University, University of Alabama at Birmingham, University of California, San Francisco, University of Washington, University of California, San Diego, Fenway Community Health Center of Harvard University, University of North Carolina, and Johns Hopkins University. We would also like to thank Drs Ronald Mitsuyasu and Jeannette Lee for their assistance with this project. Particularly, the authors would like to acknowledge Donna Porter and Dawn Grill for their significant contributions to the CNICS.

\section{References}

1. Palella FJ Jr, Delaney KM, Moorman AC, Loveless MO, Fuhrer J, Satten GA, et al. Declining morbidity and mortality among patients with advanced human immunodeficiency virus infection. HIV Outpatient Study Investigators. N Engl J Med. 1998; 338:853-860. [PubMed: 9516219]

2. Mocroft A, Ledergerber B, Katlama C, Kirk O, Reiss P, d'Arminio Monforte A, et al. Decline in the AIDS and death rates in the EuroSIDA study: an observational study. Lancet. 2003; 362:22-29. [PubMed: 12853195]

3. Engels EA, Biggar RJ, Hall HI, Cross H, Crutchfield A, Finch JL, et al. Cancer risk in people infected with human immunodeficiency virus in the United States. Int J Cancer. 2008; 123:187-194. [PubMed: 18435450]

4. Burgi A, Brodine S, Wegner S, Milazzo M, Wallace MR, Spooner K, et al. Incidence and risk factors for the occurrence of non-AIDS-defining cancers among human immunodeficiency virusinfected individuals. Cancer. 2005; 104:1505-1511. [PubMed: 16104038]

5. Frisch M, Biggar RJ, Engels EA, Goedert JJ. Association of cancer with AIDS-related immunosuppression in adults. JAMA. 2001; 285:1736-1745. [PubMed: 11277828]

6. Grulich AE, Wan X, Law MG, Coates M, Kaldor JM. Risk of cancer in people with AIDS. AIDS. 1999; 13:839-843. [PubMed: 10357384]

7. Herida M, Mary-Krause M, Kaphan R, Cadranel J, Poizot-Martin I, Rabaud C, et al. Incidence of non-AIDS-defining cancers before and during the highly active antiretroviral therapy era in a cohort of human immunodeficiency virus-infected patients. J Clin Oncol. 2003; 21:3447-3453. [PubMed: 12972519]

8. Patel P, Hanson DL, Sullivan PS, Novak RM, Moorman AC, Tong TC, et al. Incidence of types of cancer among HIV-infected persons compared with the general population in the United States, 1992-2003. Ann Intern Med. 2008; 148:728-736. [PubMed: 18490686]

9. Powles T, Robinson D, Stebbing J, Shamash J, Nelson M, Gazzard B, et al. Highly active antiretroviral therapy and the incidence of non-AIDS-defining cancers in people with HIV infection. J Clin Oncol. 2009; 27:884-890. [PubMed: 19114688]

10. Friis-Moller N, Sabin CA, Weber R, d'Arminio Monforte A, El-Sadr WM, Reiss P, et al. Combination antiretroviral therapy and the risk of myocardial infarction. N Engl J Med. 2003; 349:1993-2003. [PubMed: 14627784]

11. Mbulaiteye SMKE, Wabinga H, Parkin DM, Virgo P, Ochai R, Workneh M, et al. Spectrum of cancers among HIV-infected persons in Africa: the Uganda AIDS-Cancer Registry Match Study. Int J Cancer. 2006; 118:985-990. [PubMed: 16106415] 
12. Biggar RJ, Chaturvedi AK, Bhatia K, Mbulaiteye SM. Cancer risk in persons with HIV/AIDS in India: a review and future directions for research. Infect Agent Cancer. 2009; 4:4. [PubMed: 19327166]

13. Bowa K, Wood C, Chao A, Chintu C, Mudenda V, Chikwenya M. A review of the epidemiology of cancers at the University Teaching Hospital, Lusaka, Zambia. Trop Doct. 2009; 39:5-7. [PubMed: 19211410]

14. McGlashan NDHJ, Chelkowska E. Changes in the geographical and temporal patterns of cancer incidence among black gold miners working in South Africa, 1964-1996. Br J Cancer. 2003; 88:1361-1369. [PubMed: 12778062]

15. Engels EA, Pfeiffer RM, Goedert JJ, Virgo P, McNeel TS, Scoppa SM, et al. Trends in cancer risk among people with AIDS in the United States 1980-2002. AIDS. 2006; 20:1645-1654. [PubMed: 16868446]

16. Silverberg MJ, Chao C, Leyden WA, Xu L, Tang B, Horberg MA, et al. HIV infection and the risk of cancers with and without a known infectious cause. AIDS. 2009; 23:2337-2345. [PubMed: 19741479]

17. Crum-Cianflone N, Hullsiek KH, Marconi V, Weintrob A, Ganesan A, Barthel RV, et al. Trends in the incidence of cancers among HIV-infected persons and the impact of antiretroviral therapy: a 20-year cohort study. AIDS. 2009; 23:41-50. [PubMed: 19050385]

18. Shiels MS, Cole SR, Kirk GD, Poole C. A meta-analysis of the incidence of non-AIDS cancers in HIV-infected individuals. J Acquir Immune Defic Syndr. 2009; 52:611-622. [PubMed: 19770804]

19. Bedimo R, Chen RY, Accortt NA, Raper JL, Linn C, Allison JJ, et al. Trends in AIDS-defining and non-AIDS-defining malignancies among HIV-infected patients: 1989-2002. Clin Infect Dis. 2004; 39:1380-1384. [PubMed: 15494916]

20. Biggar RJ, Engels EA, Ly S, Kahn A, Schymura MJ, Sackoff J, et al. Survival after cancer diagnosis in persons with AIDS. J Acquir Immune Defic Syndr. 2005; 39:293-299. [PubMed: 15980688]

21. Hessol NA, Pipkin S, Schwarcz S, Cress RD, Bacchetti P, Scheer S. The impact of highly active antiretroviral therapy on non-AIDS-defining cancers among adults with AIDS. Am J Epidemiol. 2007; 165:1143-1153. [PubMed: 17344204]

22. Long JL, Engels EA, Moore RD, Gebo KA. Incidence and outcomes of malignancy in the HAART era in an urban cohort of HIV-infected individuals. AIDS. 2008; 22:489-496. [PubMed: 18301061]

23. Kitahata MM, Rodriguez B, Haubrich R, Boswell S, Mathews WC, Lederman MM, et al. Cohort profile: the Centers for AIDS Research Network of Integrated Clinical Systems. Int J Epidemiol. 2008; 37:948-955. [PubMed: 18263650]

24. Centers for Disease Control and Prevention (CDC). 1993 revised classification system for HIV infection and expanded surveillance case definition for AIDS among adolescents and adults. MMWR Recomm Rep. 1992; 41:1-19.

25. Kaplan EL, Meier P. Nonparametric estimation from incomplete observations. J Am Stat Assoc. 1958; 53:457-481.

26. Cox D. Regression models and life tables. J R Stat Soc B. 1972; 34:187-220.

27. Rubin, D. Multiple imputation for nonresponse in surveys. New York: Wiley; 1987.

28. Stuart E. Multiple imputation with large data sets: a case study of the Children's Mental Health Initiative. Am J Epidemiol. 2009; 169:1133-1139. [PubMed: 19318618]

29. Puoti M, Bruno R, Soriano V, Donato F, Gaeta GB, Quinzan GP, et al. Hepatocellular carcinoma in HIV-infected patients: epidemiological features, clinical presentation and outcome. AIDS. 2004; 18:2285-2293. [PubMed: 15577541]

30. Brau N, Fox RK, Xiao P, Marks K, Naqvi Z, Taylor LE, et al. Presentation and outcome of hepatocellular carcinoma in HIV-infected patients: a U.S.-Canadian multicenter study. J Hepatol. 2007; 47:527-537. [PubMed: 17692986]

31. Bohlius J, Schmidlin K, Costagliola D, Fatkenheuer G, May M, Caro Murillo AM, et al. Prognosis of HIV-associated non-Hodgkin lymphoma in patients starting combination antiretroviral therapy. AIDS. 2009; 23:2029-2037. [PubMed: 19531926] 
32. Chao C, Xu L, Abrams D, Leyden W, Horberg M, Towner W, et al. Survival of non-Hodgkin lymphoma patients with and without HIV infection in the era of combined antiretroviral therapy. AIDS. 2010; 24:1765-1770. [PubMed: 20453630]

33. Florence E, Lundgren J, Dreezen C, Fisher M, Kirk O, Blaxhult A, et al. Factors associated with a reduced CD4 lymphocyte count response to HAART despite full viral suppression in the EuroSIDA study. HIV Med. 2003; 4:255-262. [PubMed: 12859325]

34. Moore DM, Hogg RS, Yip B, Wood E, Tyndall M, Braitstein P, et al. Discordant immunologic and virologic responses to highly active antiretroviral therapy are associated with increased mortality and poor adherence to therapy. J Acquir Immune Defic Syndr. 2005; 40:288-293. [PubMed: 16249702]

35. Moore RD, Keruly JC. CD4R cell count 6 years after commencement of highly active antiretroviral therapy in persons with sustained virologic suppression. Clin Infect Dis. 2007; 44:441-446. [PubMed: 17205456]

36. Kelley CF, Kitchen CMR, Hunt PW, Rodriguez B, Hecht FM, Kitahata M, et al. Incomplete peripheral CD4R cell count restoration in HIV-infected patients receiving long-term antiretroviral treatment. Clin Infect Dis. 2009; 48:787-794. [PubMed: 19193107]

37. Kuller LH, Tracy R, Belloso W, De Wit S, Drummond F, Lane HC, et al. Inflammatory and coagulation biomarkers and mortality in patients with HIV infection. PLoS Med. 2008; 5:e203. [PubMed: 18942885] 
(a)

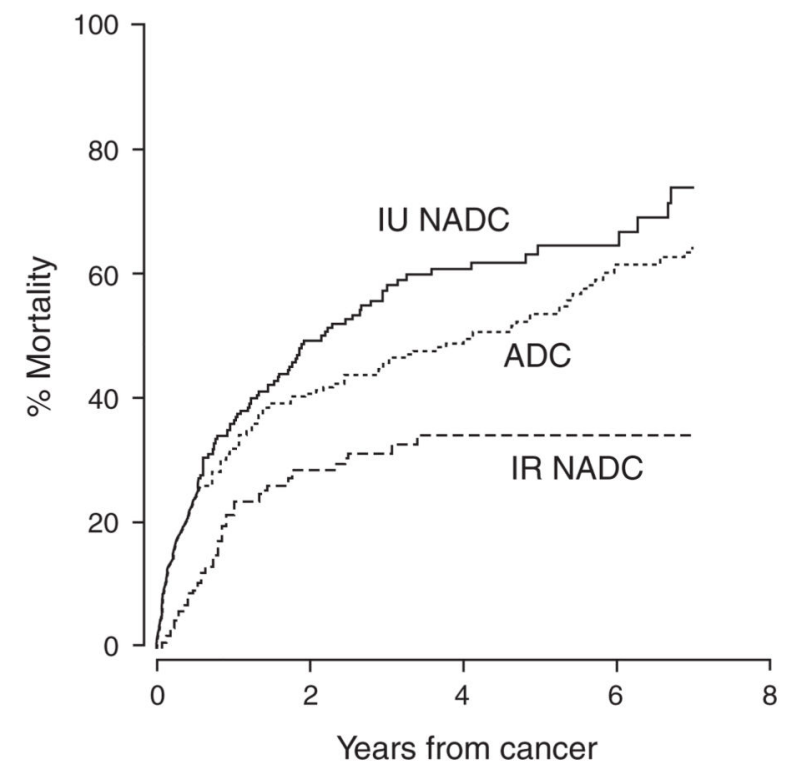

(c)

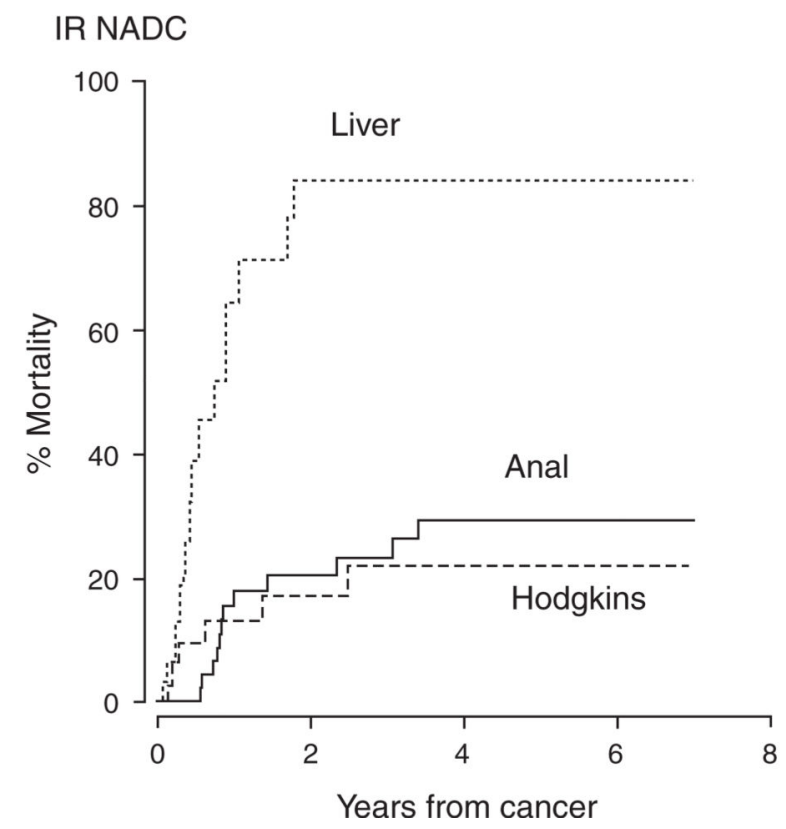

(b)

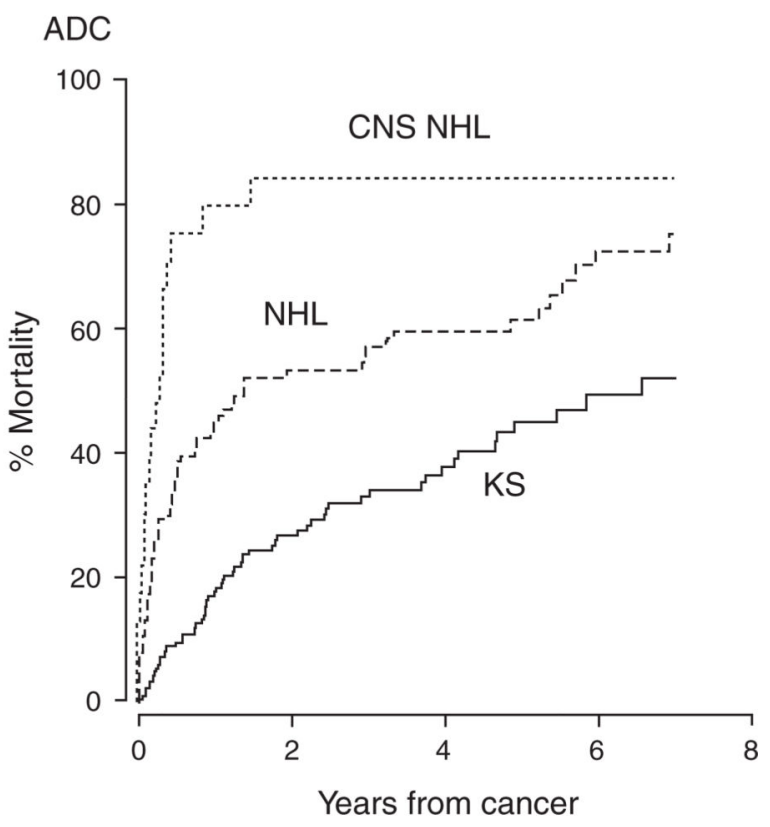

(d)

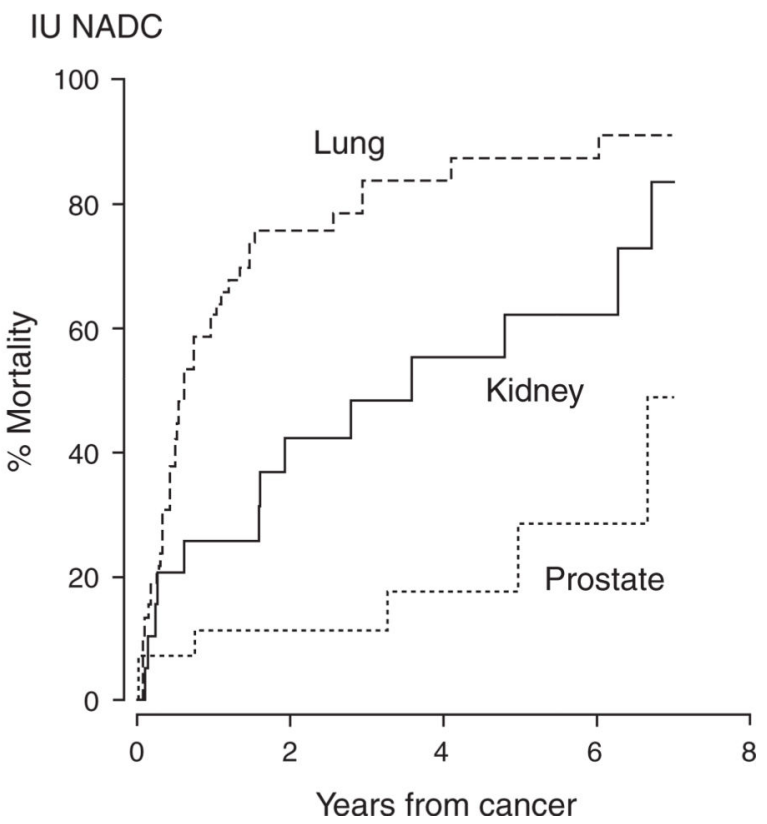

Fig. 1. Cumulative mortality over time

(a) By summary cancer type. (b-d) By individual cancer types within the cancer summary categories. ADC, AIDS-defining cancer; CNS, central nervous system; IR NADC, infectionrelated non-AIDS-defining cancer; IU NADC, infection-unrelated non-AIDS-defining cancer; KS, Kaposi's sarcoma; NHL, non-Hodgkin's lymphoma. 


\section{Table 1}

Characteristics of $650 \mathrm{HIV}$-infected adults treated with combination antiretroviral therapy in the Centers for AIDS Research Network of Integrated Clinical Systems cohort diagnosed with cancer between 1996 and 2009.

\begin{tabular}{|c|c|c|c|}
\hline Characteristics at cancer diagnosis $a$ & Survivors & Deaths & Overall \\
\hline Total, $n$ & 345 & 305 & 650 \\
\hline Follow-up time (years) & $2.8(1.2,5.0)$ & $0.6(0.2,1.5)$ & $1.4(0.4,3.7)$ \\
\hline Age (years) & $43(38,50)$ & $46(40,54)$ & $44(39,51)$ \\
\hline White, $n(\%)$ & $203(59)$ & $137(45)$ & $340(52)$ \\
\hline Male, $n(\%)$ & $293(85)$ & $263(86)$ & $556(86)$ \\
\hline HBV/HCV infection, $n(\%)$ & $60(17)$ & $74(24)$ & $134(21)$ \\
\hline IDU, $n(\%)$ & $49(14)$ & $69(23)$ & $118(18)$ \\
\hline \multicolumn{4}{|l|}{ Smoking, $n(\%)^{b}$} \\
\hline Never & $133(39)$ & $117(38)$ & $250(38)$ \\
\hline Former & $83(24)$ & $70(23)$ & $153(24)$ \\
\hline Current & $127(37)$ & $118(39)$ & $245(38)$ \\
\hline \multicolumn{4}{|l|}{ Alcohol abuse, $n(\%)^{b}$} \\
\hline Never & $270(78)$ & $226(74)$ & $496(76)$ \\
\hline Former & $25(7)$ & $29(10)$ & $54(8)$ \\
\hline Current & $46(13)$ & $50(16)$ & $96(15)$ \\
\hline Noncancer AIDS diagnosis, $n(\%)^{c}$ & $149(43)$ & $177(58)$ & $326(50)$ \\
\hline Nadir CD4 cell counts $\left(\right.$ cells $\left./ \mu \mathrm{l}^{b}\right)$ & $62(11,174)$ & $30(4,106)$ & $45(7,137)$ \\
\hline Pre-cART HIV-RNA $\left(\log _{10}\right.$ copies $\left./ \mathrm{ml}^{b}\right)$ & $5.3(4.8,5.7)$ & $5.5(4.9,5.8)$ & $5.4(4.8,5.8)$ \\
\hline Time on cART, years & $2.7(0.5,6.4)$ & $3.4(1.3,5.6)$ & $3.2(0.7,6.2)$ \\
\hline HIV-RNA suppression to $\leq 400$ copies $/ \mathrm{ml}$ & $329(95)$ & $222(73)$ & $551(85)$ \\
\hline CD4 cell count at cancer (cells $\left./ \mu^{b}\right)$ & $238(95,469)$ & $154(23,308)$ & $204(55,393)$ \\
\hline HIV-RNA at cancer $\left(\log _{10}\right.$ copies $\left./ \mathrm{ml}^{b}\right)$ & $2.3(1.4,4.4)$ & $3.1(1.9,4.8)$ & $2.6(1.4,4.6)$ \\
\hline
\end{tabular}

cART, combination antiretroviral therapy; CDC, Centers for Disease Control and Prevention; HBV, hepatitis B virus; HCV, hepatitis C virus; IDU, intravenous drug use; KS, Kaposi's sarcoma; NHL, non-Hodgkin's lymphoma.

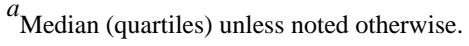

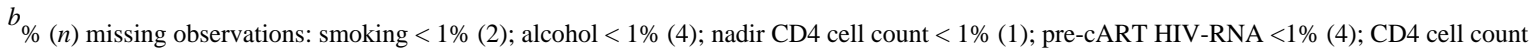
at cancer $=7 \%(44)$; HIV-RNA at cancer $=8 \%(51)$.

${ }^{c}$ A noncancer AIDS diagnosis included any CDC AIDS-defining illness with the exception of NHL, KS or cervical cancer at or before cancer diagnosis. 
Table 2

Characteristics of 650 incident cancer cases among HIV-infected adults treated with combination antiretroviral therapy in the Centers for AIDS Research Network of Integrated Clinical Systems cohort between 1996 and 2009.

\begin{tabular}{|c|c|c|c|}
\hline Characteristics at cancer diagnosis $a$ & Survivors & Deaths & Overall \\
\hline Total, $n$ & 345 & 305 & 650 \\
\hline Median year of diagnosis (range) & $6 / 05(2 / 03 ; 2 / 07)$ & $7 / 03(5 / 01 ; 1 / 06)$ & $6 / 04(2 / 02 ; 9 / 06)$ \\
\hline Cancer diagnosis & $n(\%)$ & $n(\%)$ & $n(\%)$ \\
\hline KS & $123(36)$ & $62(20)$ & $185(29)$ \\
\hline Non-CNS NHL & $43(12)$ & $71(23)$ & $114(18)$ \\
\hline Lung & $11(3)$ & $48(16)$ & $59(9)$ \\
\hline Anal & $40(12)$ & $15(5)$ & $55(8)$ \\
\hline Hodgkin's lymphoma & $26(7)$ & $6(2)$ & $32(5)$ \\
\hline Prostate & $21(6)$ & $6(2)$ & $27(4)$ \\
\hline CNS NHL & $3(1)$ & $19(6)$ & $22(3)$ \\
\hline Liver & $3(1)$ & $19(6)$ & $22(3)$ \\
\hline Kidney & $6(2)$ & $13(4)$ & $19(3)$ \\
\hline Melanoma & $13(4)$ & $2(1)$ & $15(2)$ \\
\hline Breast & $8(2)$ & $3(1)$ & $11(2)$ \\
\hline Colorectal & $7(2)$ & $4(1)$ & $11(2)$ \\
\hline Other cancers $b$ & $41(12)$ & $37(13)$ & $78(12)$ \\
\hline \multicolumn{4}{|l|}{ Summary cancer diagnosis } \\
\hline Infection-unrelated NADCs & $99(29)$ & $122(40)$ & $221(34)$ \\
\hline $\mathrm{ADCs}^{c}$ & $169(49)$ & $152(50)$ & $321(49)$ \\
\hline Infection-related NADCs $d$ & $77(22)$ & $31(10)$ & $108(17)$ \\
\hline \multicolumn{4}{|l|}{ Summary stage } \\
\hline I & $38(11)$ & $13(4)$ & $51(8)$ \\
\hline II & $27(8)$ & $21(7)$ & $48(7)$ \\
\hline III & $17(5)$ & $21(7)$ & $38(6)$ \\
\hline IV & $43(12)$ & $76(25)$ & $119(19)$ \\
\hline Missing & $86(25)$ & $85(28)$ & $171(26)$ \\
\hline Not applicable $e^{e}$ & $134(39)$ & $89(29)$ & $223(34)$ \\
\hline 'Stage IV equivalent', $n(\%) f$ & $98(39)$ & $139(66)$ & $237(52)$ \\
\hline Biopsy confirmation, $n(\%)^{g}$ & $280(81)$ & $262(86)$ & $542(83)$ \\
\hline Cancer treatment, $n(\%)^{h}$ & $270(78)$ & $215(71)$ & $485(75)$ \\
\hline
\end{tabular}

ADC, AIDS-defining cancer; CNS, central nervous system; KS, Kaposi's sarcoma; NADC, non-AIDS-defining cancer; NHL, non-Hodgkin's lymphoma.

${ }^{a}$ Median (quartiles) unless noted otherwise.

Other cancers: cervical, biliary, bladder/urinary, primary brain, esophagus, oral cavity/pharynx, other head and neck, leukemia, multiple myeloma, other (not specified or unknown origin), ovary, pancreas, penis, peritoneum/retroperitoneum, small intestine, soft tissue, stomach, testicular, thyroid, trachea/pleura, uterus, and vagina/vulva. 
${ }^{c}$ ADCs: cervical, KS, and NHL.

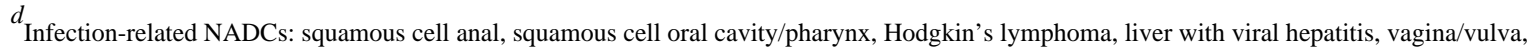
and penis.

${ }^{e}$ Cancers that do not use I-IV summary staging: KS, primary CNS NHL, leukemia, multiple myeloma, and primary brain.

$f_{\text {Among }} 463$ cases (251 survivors and 212 deaths) that could be classified as 'stage IV equivalent' (excluding 171 missing and 16 cases of leukemia, multiple myeloma, and primary brain). Summary stage IV, visceral KS and primary CNS NHL classified as 'stage IV equivalent.' Summary stages I to III and mucocutaneous KS was classified as 'not stage IV equivalent;'.

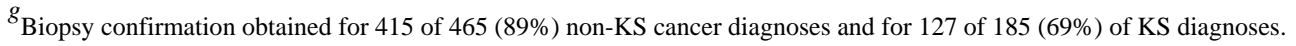

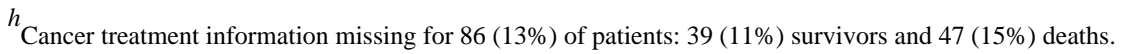




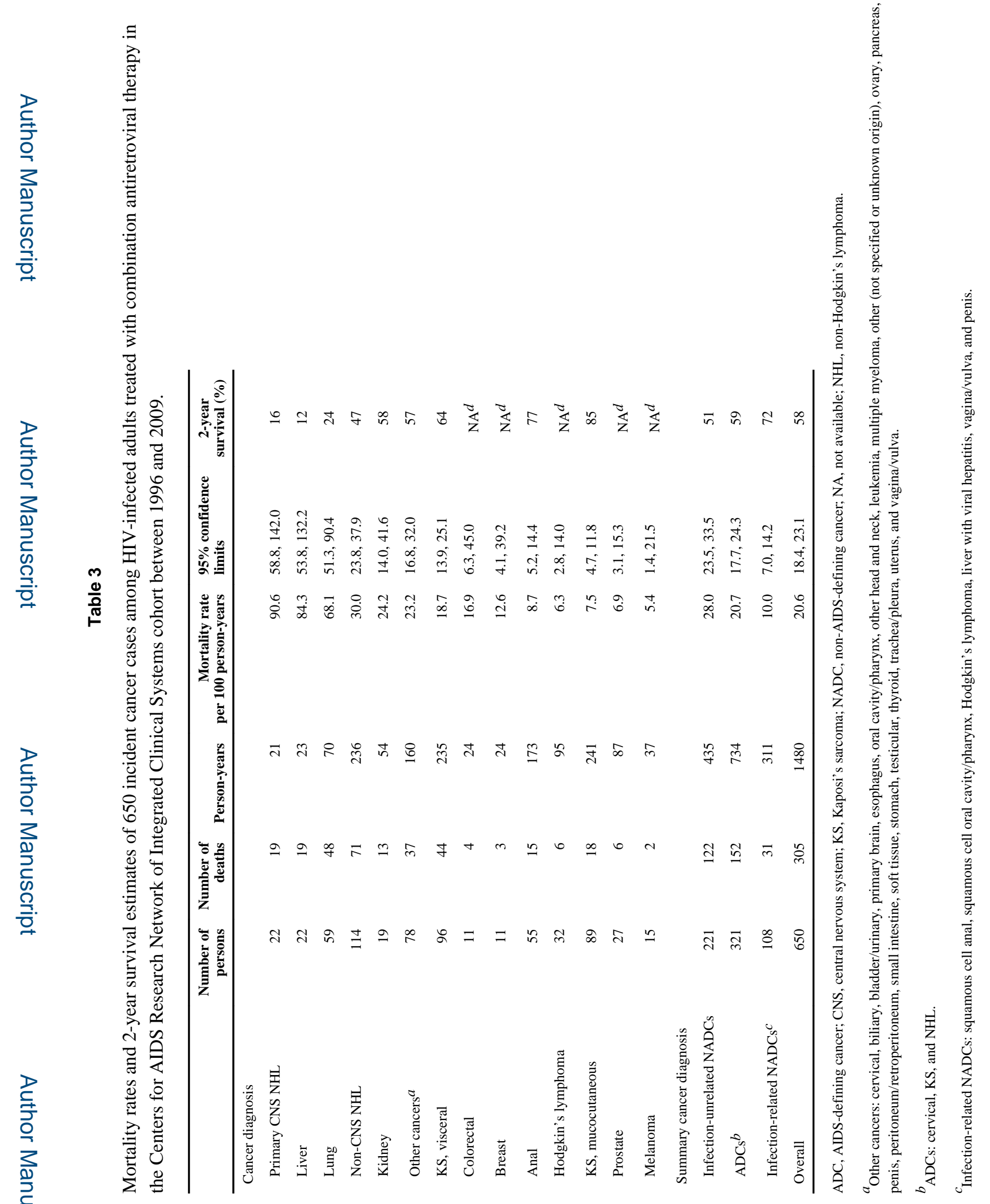




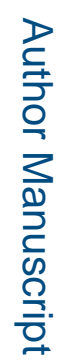

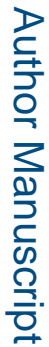

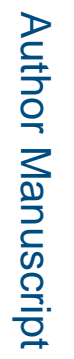

AIDS. Author manuscript; available in PMC 2015 March 31. 
Table 4

Adjusted mortality rate ratios of 650 incident cancer cases among HIV-infected adults treated with combination antiretroviral therapy in the Centers for AIDS Research Network of Integrated Clinical Systems cohort between 1996 and 2009.

\begin{tabular}{|c|c|c|c|}
\hline & Adjusted hazard ratio $a$ & $95 \%$ confidence intervals & $P$-value \\
\hline Age, per decade & 1.44 & $1.25,1.66$ & $<0.01$ \\
\hline Nonwhite vs. white & 1.24 & $0.97,1.59$ & 0.09 \\
\hline Male vs. female & 1.05 & $0.73,1.51$ & 0.78 \\
\hline $\mathrm{HBV} / \mathrm{HCV}$ infection & 1.29 & $0.97,1.70$ & 0.08 \\
\hline \multicolumn{4}{|l|}{ Smoking } \\
\hline Current vs. never & 1.00 & $0.76,1.32$ & 0.99 \\
\hline Past vs. never & 0.77 & $0.56,1.10$ & 0.11 \\
\hline \multicolumn{4}{|l|}{ Alcohol abuse } \\
\hline Current vs. never & 0.96 & $0.70,1.32$ & 0.81 \\
\hline Past vs. never & 1.30 & $0.86,1.95$ & 0.22 \\
\hline Nadir CD4 cell count, per 100 cells/ $\mu 1$ & 0.99 & $0.85,1.15$ & 0.85 \\
\hline $\mathrm{CD} 4$ cell count at cancer, per 100 cells $/ \mu \mathrm{l}$ & 0.90 & $0.83,0.98$ & 0.01 \\
\hline HIV-RNA suppression to $\leq 400$ copies/ml & 0.32 & $0.23,0.44$ & $<0.01$ \\
\hline Pre-cART HIV-RNA, per $\log _{10}$ & 1.00 & $0.86,1.15$ & 0.97 \\
\hline Noncancer AIDS diagnosis & 1.21 & $0.94,1.55$ & 0.14 \\
\hline 'Stage IV equivalent' $b$ & 2.30 & $1.69,3.13$ & $<0.01$ \\
\hline Cancer treatment & 0.55 & $0.39,0.79$ & $<0.01$ \\
\hline Date of cancer diagnosis, per year & 0.99 & $0.95,1.03$ & 0.57 \\
\hline \multicolumn{4}{|l|}{ Summary cancer diagnosis } \\
\hline Infection-unrelated NADCs & 1 & Reference & \\
\hline $\mathrm{ADCs}^{c}$ & 0.59 & $0.42,0.81$ & $<0.01$ \\
\hline Infection-related NADCs $d$ & 0.52 & $0.34,0.78$ & $<0.01$ \\
\hline
\end{tabular}

ADC, AIDS-defining cancer; cART, combination antiretroviral therapy; CNS, central nervous system; HBV, hepatitis B virus; HCV, hepatitis C virus; KS, Kaposi's sarcoma; NADC, non-AIDS-defining cancer; NHL, non-Hodgkin's lymphoma.

${ }^{a}$ Adjusted for all variables in the table.

${ }^{b}$ Summary stage IV, visceral KS, and primary CNS NHL classified as 'stage IV equivalent.' Summary stage I-III and mucocutaneous KS classified as 'not stage IV equivalent.' In the final model, 'stage IV equivalent' was imputed for 187 of 650 (29\%) cases with missing information.

${ }^{c}$ ADCs: cervical, KS, and NHL.

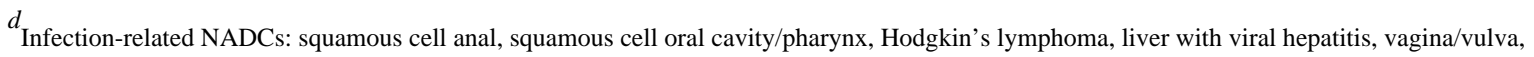
and penis. 